\title{
Trajectory generation for continuous leg forces during double support and heel-to-toe shift based on divergent component of motion
}

\author{
Johannes Englsberger ${ }^{\dagger}$, Twan Koolen ${ }^{\ddagger *}$, Sylvain Bertrand ${ }^{\ddagger}$, Jerry Pratt ${ }^{\ddagger}$, Christian Ott $^{\dagger}$, Alin Albu-Schäffer ${ }^{\dagger}$
}

\begin{abstract}
This paper works with the concept of Divergent Component of Motion (DCM), also called '(instantaneous) Capture Point'. We present two real-time DCM trajectory generators for uneven (three-dimensional) ground surfaces, which lead to continuous leg (and corresponding ground reaction) force profiles and facilitate the use of toe-off motion during double support. Thus, the resulting DCM trajectories are well suited for real-world robots and allow for increased step length and step height. The performance of the proposed methods was tested in numerous simulations and experiments on IHMC's Atlas robot and DLR's humanoid robot TORO.
\end{abstract}

\section{INTRODUCTION}

For humanoid robots to be used in real world scenarios, there is a need of robust and simple walking controllers. Many successful Linear Inverted Pendulum (LIP) based walking control methods have been presented recently [1][6]. Wieber [3] and Herdt et al. [4] propose trajectory free approaches for planning based on the solution of a Quadratic Program (QP) in a Model Predictive Control (MPC) framework. The use of the LIP model for bipedal walking control is typically restricted to horizontal motions of the CoM $(z=$ const.). This motivates the derivation of methods for non-constant CoM and floor height. Kajita et al. [7] introduce the 3D Linear Inverted Pendulum Mode, which constrains the CoM to be on a (not necessarily horizontal) plane. They present experiments for walking on spiral stairs, yet a rather static walking is achieved. Zhao and Sentis [8] present a method for three-dimensional foot placement planning on uneven ground surfaces based on the Prismatic Inverted Pendulum. Yet, the lateral foot-placement cannot be predefined, but is dependent on the sagittal dynamics and the "desired CoM Surface". Additionally, the method is restricted to ground surfaces with laterally constant heights.

To simplify the design of walking controllers, several previous works, such as [1], [11]-[16], propose to split the CoM dynamics into a stable and an unstable part. All these methods are based on LIP model dynamics and thus most of them are theoretically restricted to walking on flat ground. In [17] we overcame the limitation of constant CoM and floor height by extending the concept of Divergent Component of Motion (DCM [1], also called '(instantaneous) Capture

\footnotetext{
$\dagger$ German Aerospace Center (DLR), Institute of Robotics and Mechatronics, 82234 Wessling, Germany. contact: johannes .englsberger@dlr.de

$¥$ Florida Institute for Human and Machine Cognition (IHMC)

* Massachusetts Institute of Technology (MIT), Computer Science and Artificial Intelligence Laboratory
}

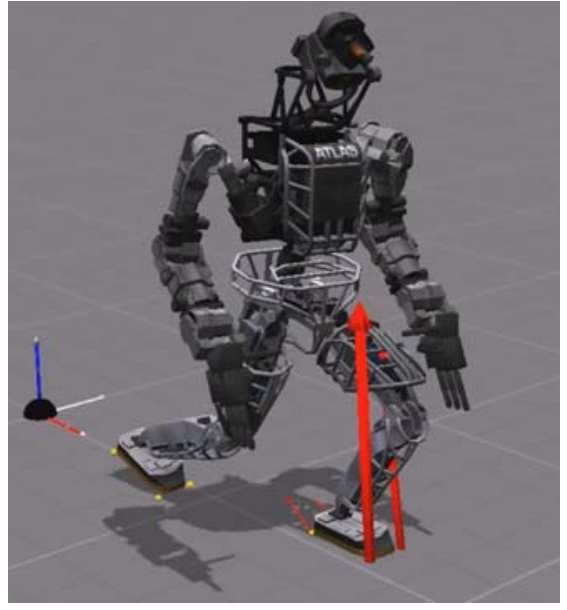

Fig. 1. Atlas robot [9] doing $70 \mathrm{~cm}$ steps in IHMC's simulation environment (SimulationConstructionSet [10]) using toe-off motion.

Point' [18]) to 3D. Therefore, the "Enhanced Centroidal Moment Pivot point" (eCMP) and the "Virtual Repellent Point" (VRP) were introduced, which allow for the encoding of both direction and magnitude of the external (e.g. leg) forces and the total force (i.e. external forces plus gravity) acting on the robot. Based on eCMP, VRP and DCM, a method for real-time planning and control of DCM trajectories in $3 \mathrm{D}$ was presented. Yet, the DCM trajectory generator presented in [17] assumed constant eCMP positions with instantaneous transitions between subsequent single support phases (no double support considered). This lead to discontinuous desired leg forces and desired joint torques, which can cause perturbations in the actuation system. Therefore, as the first contribution of this paper, a method for generating Continuous Double Support (CDS) trajectories is introduced, which - similar to the "Multi-Contact Transitions" in [8]results in smooth eCMP and related leg force profiles.

The use of toe-off motion facilitates energy efficient [19] and human-like [20] walking. Thus, the second contribution of this paper is the extension of the CDS trajectory generator to a Heel-to-Toe (HT) trajectory generator, in which the eCMP is shifted from heel to toe during single support. This allows for early toe-off motions during double support, increasing maximum achievable step length and step height. Throughout the paper we will often use the terms "eCMP" (and its corresponding "VRP") and "DCM". For certain assumptions (constant CoM height, constant angular momen- 


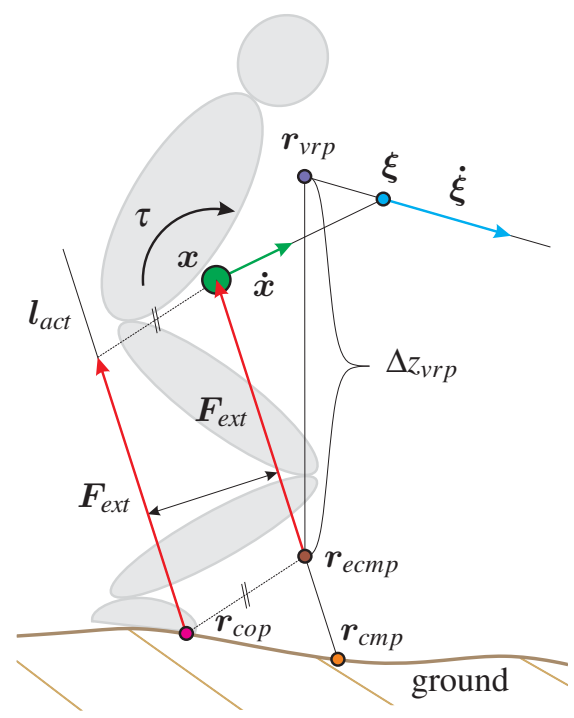

Fig. 2. Point correlations for general robot dynamics. The CMP is found as intersection of the line CoM-to-eCMP with the ground. The line of action $\boldsymbol{l}_{\text {act }}$ of the external force can be shifted via a torque $\tau$ around the CoM $(\dot{\boldsymbol{L}} \neq 0)$, so that the CoP does generally not coincide with the CMP.

tum w.r.t. CoM [21]), the eCMP can be equivalent to the ZMP [22] and the DCM to the (instantaneous) Capture Point (iCP, [18]), so many ideas presented here can be applied to ZMP- and iCP-based methods.

The paper is organized as follows: In Section II, we recapitulate the main results from [17] as the derivations in this paper build on the methods proposed there. Section III contains the main theoretical contributions of the paper, namely the derivation of DCM reference trajectories for continuous double support (CDS) and heel-to-toe (HT) transfer. Section IV outlines the used walking state machine. Section $\mathrm{V}$ discusses the trajectories resulting from the proposed trajectory generators. In section VI, we outline the simulations and experiments that were performed to validate the proposed methods. Section VII concludes the paper.

\section{BACKGROUND [17]}

\section{A. Introduction of three-dim. DCM, eCMP and VRP}

Motivated by the performance of 2D Capture Point (= DCM) control [13], [14], the three-dimensional Divergent Component of Motion (DCM) was introduced as

$$
\xi=\boldsymbol{x}+b \dot{\boldsymbol{x}},
$$

where $\boldsymbol{\xi}=\left[\boldsymbol{\xi}_{x}, \boldsymbol{\xi}_{y}, \boldsymbol{\xi}_{z}\right]^{T}$ is the DCM, $\boldsymbol{x}=[x, y, z]^{T}$ and $\dot{\boldsymbol{x}}=[\dot{x}, \dot{y}, \dot{z}]^{T}$ are the CoM position and velocity and $b>0$ is the time-constant of the DCM dynamics. By reordering (1), the CoM dynamics is found as

$$
\dot{\boldsymbol{x}}=-\frac{1}{b}(\boldsymbol{x}-\boldsymbol{\xi})
$$

This shows that the CoM has a stable first order dynamics for $b>0$ ( $\rightarrow$ it follows the DCM). Additionally, the so

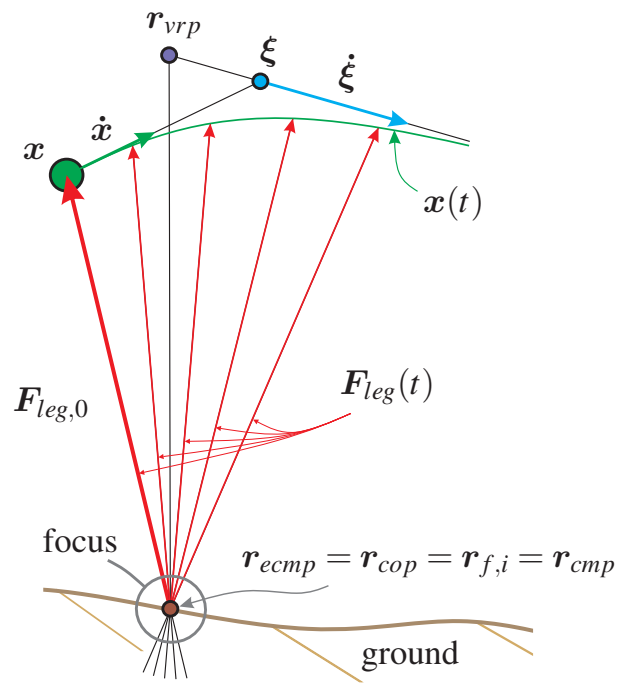

Fig. 3. For planning, the eCMP is designed to coincide with the point foot (or foot center) which along with the assumption $\dot{\boldsymbol{L}}=0$ leads to a constant focus point, through which all force lines pass.

called Enhanced Centroidal Moment Pivot point (eCMP) was introduced, which encodes the external (e.g. leg-) forces in a simple repelling force law (linear dependency), based on the difference of the CoM and the eCMP:

$$
\boldsymbol{F}_{\text {ext }}=\frac{m}{b^{2}}\left(\boldsymbol{x}-\boldsymbol{r}_{e c m p}\right)
$$

The eCMP is closely related to the CMP [21], but it is not restricted to be within the foot plane or ground surface. This allows for encoding of not only the direction of the total external force, but also its magnitude. To encode the sum of all forces acting on the CoM including gravity, the Virtual Repellent Point (VRP) was introduced as

$$
\boldsymbol{r}_{v r p}=\boldsymbol{r}_{e c m p}+\left[\begin{array}{lll}
0 & 0 & b^{2}
\end{array}\right]^{T}=\boldsymbol{r}_{e c m p}+\left[\begin{array}{lll}
0 & 0 & \Delta z_{v r p}
\end{array}\right]^{T} .
$$

This leads to the following DCM dynamics:

$$
\dot{\xi}=\frac{1}{b}\left(\boldsymbol{\xi}-\boldsymbol{r}_{v r p}\right)
$$

This shows that the DCM has an unstable first order dynamics ("pushed" by the VRP on a straight line). The VRP encodes gravity and external forces in a single point:

$$
\boldsymbol{F}=\frac{m}{b^{2}}\left(\boldsymbol{x}-\boldsymbol{r}_{v r p}\right) .
$$

Figure 2 clarifies the correlations between the eCMP, CMP and $\mathrm{CoP}$ for a general (bipedal) robot dynamics.

\section{B. Generation of DCM reference}

The basic idea in [17] - exploiting the first order DCM dynamics - was to find a DCM trajectory which corresponds to constant eCMPs in the centers of the preplanned future foot positions $\boldsymbol{r}_{f, i}$, thus fulfilling the ZMP constraints. Given 


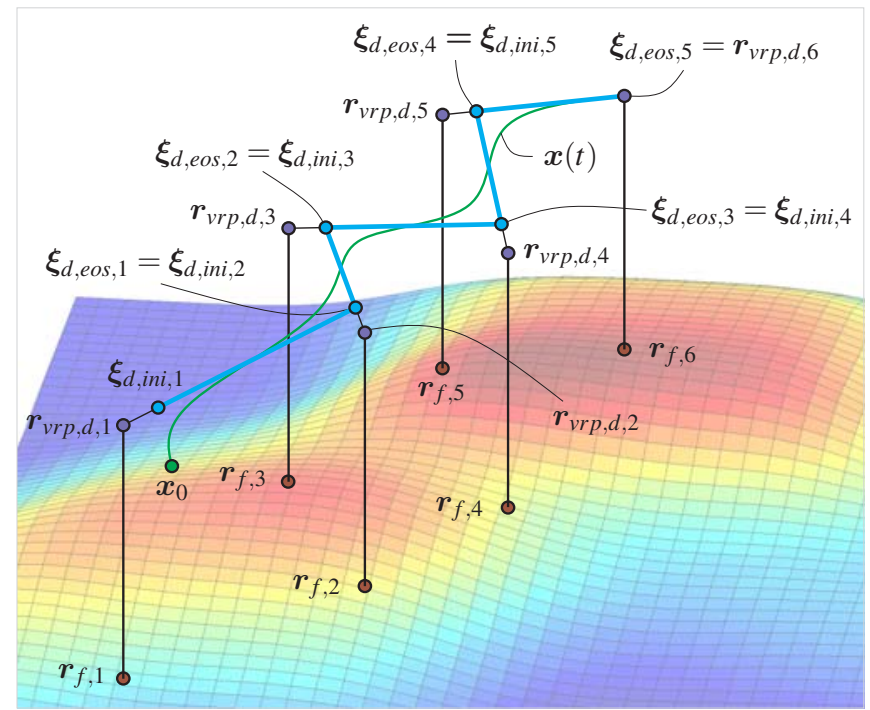

Fig. 4. Planning of DCM trajectory over rough terrain. The DCM reference trajectory (bold lines) and the resulting CoM trajectory (sinusoidal curve, "automatically" follows the DCM) are three-dimensional.

a desired eCMP-to-VRP height difference $\Delta z_{v r p}$, the according desired VRPs (see Fig. 4) are found via (4) as

$$
\boldsymbol{r}_{v r p, d, i}=\boldsymbol{r}_{f, i}+\left[\begin{array}{lll}
0 & 0 & \Delta z_{v r p}
\end{array}\right]^{T}
$$

The time-constant of the DCM dynamics results from (4) as $b=\sqrt{\Delta z_{v r p} / g}$. The VRP-height $\Delta z_{v r p}$ can be intuitively interpreted as an average height of the CoM over the ground surface. The desired DCM locations at the end of each step are found via recursion:

$$
\boldsymbol{\xi}_{d, e o s, i-1}=\boldsymbol{\xi}_{d, i n i, i}=\boldsymbol{r}_{v r p, d, i}+e^{-\frac{t_{s t e p}}{b}}\left(\boldsymbol{\xi}_{d, e o s, i}-\boldsymbol{r}_{v r p, d, i}\right) .
$$

For $t<t_{\text {step }}$, the desired DCM trajectory in time is

$$
\boldsymbol{\xi}_{d}(t)=\boldsymbol{r}_{v r p, d, 1}+e^{\frac{t-t_{s t e p}}{b}}\left(\boldsymbol{\xi}_{d, e o s, 1}-\boldsymbol{r}_{v r p, d, 1}\right) .
$$

\section{Three-dimensional DCM tracking control}

The DCM control law used in this work is given by

$$
\boldsymbol{r}_{v r p, c}=\boldsymbol{\xi}+k_{\xi} b\left(\boldsymbol{\xi}-\boldsymbol{\xi}_{\boldsymbol{d}}\right)-b \dot{\boldsymbol{\xi}}_{d} .
$$

It leads to the following stable closed loop dynamics

$$
\left[\begin{array}{c}
\dot{\boldsymbol{x}} \\
\dot{\boldsymbol{\xi}}
\end{array}\right]=\underbrace{\left[\begin{array}{cc}
-1 / b & 1 / b \\
0 & -k_{\xi}
\end{array}\right]\left[\begin{array}{l}
\boldsymbol{x} \\
\boldsymbol{\xi}
\end{array}\right]}_{\text {feedback }}+\underbrace{\left[\begin{array}{cc}
0 & 0 \\
k_{\xi} & 1
\end{array}\right]\left[\begin{array}{l}
\boldsymbol{\xi}_{d} \\
\dot{\boldsymbol{\xi}}_{d}
\end{array}\right]}_{\text {feedforward }} .
$$

As the DCM error $\boldsymbol{e}_{\boldsymbol{\xi}}=\boldsymbol{\xi}-\boldsymbol{\xi}_{d}$ converges asymptotically, also the commanded VRP $\boldsymbol{r}_{v r p, c}$ and its corresponding eCMP $\boldsymbol{r}_{e c m p, c}$ converge to their desired values $\left(\boldsymbol{r}_{v r p, d, 1}\right.$ and $\boldsymbol{r}_{e c m p, d, 1}=\boldsymbol{r}_{f, 1}$ ) asymptotically after a perturbation. The according desired external force can be found as

$$
\boldsymbol{F}_{\text {ext }, c}=\frac{m g}{\Delta z_{\text {vrp }}}\left(\boldsymbol{x}-(\underbrace{\boldsymbol{r}_{v r p, c}-\left[\begin{array}{lll}
0 & 0 & \Delta z_{v r p}
\end{array}\right]^{T}}_{\boldsymbol{r}_{\text {ecm }, c}})\right)
$$

Note that the only equations that are finally needed are (8) and (9) for 3D DCM trajectory generation and (10) and (12) for force-based DCM tracking control. They can easily be computed in real-time on any computer. The design parameters are the VRP-height $\Delta z_{v r p}$, the DCM control gain $k_{\xi}$ and the time per step $t_{\text {step }}$.

\section{Continuous Double Support (CDS) AND HEEL-TO-TOE (HT) TRAJECTORIES}

\section{A. Continuous Double Support trajectories}

The planning method presented in [17] (and recapitulated in section II-B) is very powerful, as it allows for the design of walking trajectories over unstructured (3D) ground surfaces in real-time. Yet, as the original method only considers single support phases (and instantaneous transitions between them), there is the drawback of discontinuous desired VRP (and eCMP) and thus discontinuous desired leg forces (and ground reaction forces, respectively) at the support transitions (see $F_{\text {ext }}$ in Fig. 8). This leads to considerable discontinuities in the commanded joint torques, which can be unfeasible for a physical robot due to its limited actuator dynamics and might excite unmodeled structural elasticities. This motivates the derivation of DCM trajectories which satisfy $\mathrm{C} 1$ continuity and thus lead to continuous eCMP (and corresponding leg force) transitions. Therefore, we will make use of a walking state machine (described in Sec. IV) and explicitly consider the anticipated double support phases for the generation of a modified DCM reference trajectory.

Figure 5 (left) shows the outline used for the generation of DCM trajectories with continuous double support (CDS) transitions. The CDS trajectory generation builds on the method proposed in [17] (based on eCMPs in the foot centers and instantaneous single support transitions), which leads to the zigzag trajectory for the DCM shown in Fig. 4 (bright blue lines). The edges around the initial DCMs $\boldsymbol{\xi}_{\text {ini,i }}$ are now "rounded" (turquoise curves) to guarantee continuity of the eCMP and corresponding leg forces during double support. For a desired DCM position and velocity, the correlating VRP is obtained from (5) as

$$
\boldsymbol{r}_{v r p}=\boldsymbol{\xi}-b \dot{\boldsymbol{\xi}}
$$

This means that a reference trajectory with continuous developing of DCM position and velocity (C1 continuity) will result in a continuous VRP trajectory and corresponding continuous eCMP (see (4)) and leg force (see (3)) trajectories. This motivates the use of a third order polynomial interpolation to "round" the edges of the preliminary DCM reference trajectory (corresponding to smooth transition during double support). Given a desired double support duration $t_{D S}$, the idea is to compute two points

$$
\boldsymbol{\xi}_{\text {iniDS }, i}=\boldsymbol{r}_{v r p, i-1}+e^{-\frac{\Delta t_{D S, i n i}}{b}}\left(\boldsymbol{\xi}_{\text {ini }, i}-\boldsymbol{r}_{v r p, i-1}\right)
$$

("iniDS" stands for "initial double support") and

$$
\boldsymbol{\xi}_{\text {eoDS }, i}=\boldsymbol{r}_{v r p, i}+e^{\frac{\Delta t_{D S, \text { end }}}{b}}\left(\boldsymbol{\xi}_{\text {ini,i }}-\boldsymbol{r}_{v r p, i}\right)
$$




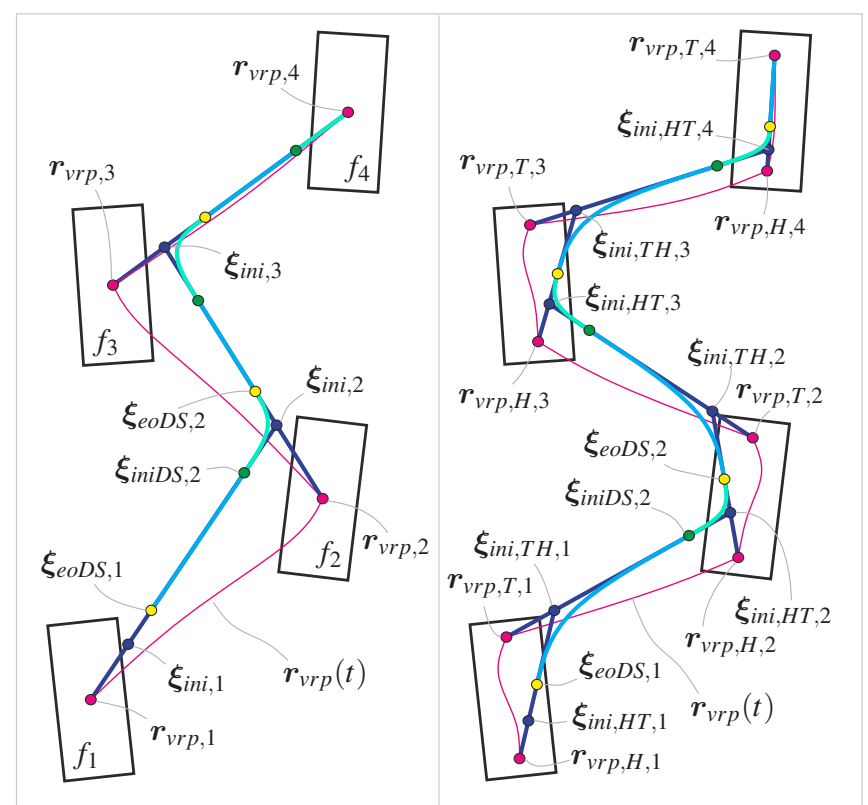

Fig. 5. Delineation of Continuous Double Support (left) and Heel-to-Toe (including continuous double support; right) DCM trajectories. Depicted for three previewed future footsteps.

("eoDS" stands for "end of double support") on the preliminary DCM trajectory (see Fig. 5 (left)), where the DCM would be $\Delta t_{D S, \text { ini }}=\alpha_{D S \text {,ini }} t_{D S}$ before and $\Delta t_{D S, \text { end }}=\left(1-\alpha_{D S, \text { ini }}\right) t_{D S}$ after the nominal (instantaneous) support transition. In this paper, we chose the parameter $\alpha_{D S, i n i}$ to be $0.5 . \boldsymbol{\xi}_{\text {iniDS,i }}$ and $\boldsymbol{\xi}_{e o D S, i}$ (and the corresponding DCM velocities) are used as initial and final boundary conditions for the third order polynomial, which smoothly interpolates between the two. A polynomial parameter matrix, which fulfills initial and final DCM position and velocity boundary conditions, can be computed as

$$
\boldsymbol{P}=\left[\begin{array}{cccc}
2 / T^{3} & 1 / T^{2} & -2 / T^{3} & 1 / T^{2} \\
-3 / T^{2} & -2 / T & 3 / T^{2} & -1 / T \\
0 & 1 & 0 & 0 \\
1 & 0 & 0 & 0
\end{array}\right]\left[\begin{array}{c}
\boldsymbol{\xi}_{i n i}^{T} \\
\dot{\boldsymbol{\xi}}_{\text {ini }}^{T} \\
\boldsymbol{\xi}_{\text {end }}^{T} \\
\dot{\boldsymbol{\xi}}_{\text {end }}^{T}
\end{array}\right]
$$

where $T$ denotes the total duration of the transition and $\left[\boldsymbol{\xi}_{\text {ini }}^{T}, \dot{\boldsymbol{\xi}}_{\text {ini }}^{T}, \boldsymbol{\xi}_{\text {end }}^{T}, \dot{\boldsymbol{\xi}}_{\text {end }}^{T}\right]^{T}$ are the boundary conditions on the initial and final DCM position and velocity. The column vectors in $P=\left[p_{x}, p_{y}, p_{z}\right]$ contain the polynomial parameters for $\mathrm{x}, \mathrm{y}$ and $\mathrm{z}$ direction. With $\boldsymbol{P}$, the DCM position and velocity can be computed for any time $t \in[0, T]$ as

$$
\left[\begin{array}{c}
\boldsymbol{\xi}^{T}(t) \\
\dot{\boldsymbol{\xi}}^{T}(t)
\end{array}\right]=\left[\begin{array}{cccc}
t^{3} & t^{2} & t & 1 \\
3 t^{2} & 2 t & 1 & 0
\end{array}\right] \boldsymbol{P},
$$

where $t$ denotes the time in state (see Sec. IV). This polynomial interpolation (using $\boldsymbol{\xi}_{\text {iniDS }, i}, \boldsymbol{\xi}_{e o D S, i}$ and the according DCM velocities as boundary conditions and setting $T=t_{D S}$ in (16)) is used during double support (turquoise curves in Fig. 5 (left)). The VRP, eCMP and leg-force can then be

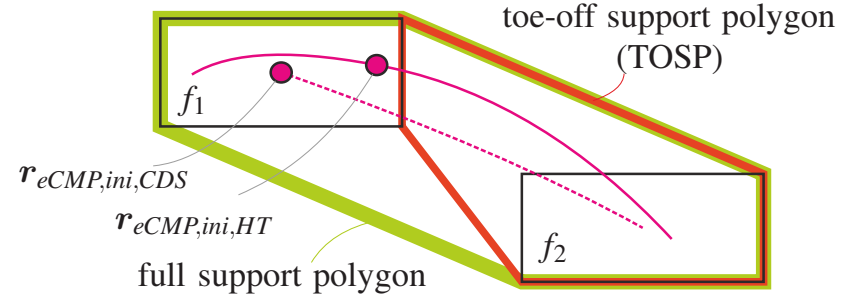

Fig. 6. Full support polygon and toe-off support polygon (TOSP)

computed via (13), (4) and (3). During single support, exponential interpolation (9) is used (bright blue line segments). Figure 5 (left) also shows the continuous VRP trajectories (pink curves) during double support. During single support, the VRP positions are constant (marked by $\boldsymbol{r}_{v r p, i}$ ). Note that Fig. 5 shows a top view of the desired trajectories. Yet, just like in Fig. 4, the proposed methods and resulting DCM, VRP and eCMP trajectories are three-dimensional in general.

Note that the use of the proposed polynomial interpolation guarantees for continuity of the commanded VRPs and corresponding eCMPs, whilst feasibility (corresponding CoP in base of support) is not guaranteed. In case of a feasibilty violation, the eCMP would have to be projected to the feasible region (e.g. as proposed in [14]). Yet, for the chosen parameter $\alpha_{D S, i n i}=0.5$ we did not encounter any feasibility violation in simulations or experiments.

\section{B. Continuous Heel-to-Toe (HT) trajectories}

The methods derived for continuous double support transitions turn out to be useful to address another problem: when being restricted to full foot support (no toe-off) during double support, the maximum step length a walking robot can achieve and the maximum stair height it can step up is limited. This is mainly due to singularities related to the stretched knee of the hind leg in the end of double support. To overcome this problem, a robot can make use of its toes or allow the rear foot to tilt around its front edge ("toe-off" motion $\rightarrow$ increase of effective hind leg length) during double support. While reducing the singularity problem, a toe-off introduces a new problem: to avoid tilting of the robot about the edges of its support polygon (for flat ground; in 3D: similar issues related to unilaterality of foot forces), the desired $\mathrm{CoP}$ has to stay inside the support polygon. During toe-off, the support polygon is instantaneously decreased to the "toe-off support polygon" (TOSP, see Fig. 6). For our DCM reference generation, we assume the eCMP to coincide with the $\mathrm{CoP}$ ( $\rightarrow$ no torques around CoM considered, see Fig. 2 and 3). Thus, if the reference eCMP lies outside the TOSP, it has to be projected to it, resulting in a discontinuous eCMP (and related leg and ground reaction forces, respectively). One possibility to avoid this problem is to wait for the eCMP reference to enter the TOSP before toe-off is initiated. In the method for continuous double support (CDS, Sec. III-A), the eCMP trajectories start from the foot-centers (see $\boldsymbol{r}_{e C M P, i n i, C D S}$ in Fig. 6), such that 


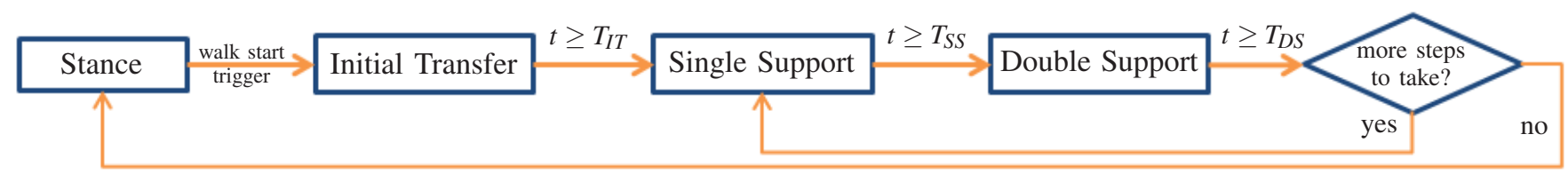

Fig. 7. Walking state machine: state $\in\left[\right.$ Stance, Initial Transfer (IT), Single Support (SS), Double Support (DS)], state durations: $T_{I T}, T_{S S}, T_{D S}$

it takes comparably long until the eCMP reaches the TOSP. The second option is to plan a DCM reference trajectory which results in an earlier entry of the corresponding eCMP in the TOSP. Here we propose a heel-to-toe shift of the eCMP during single support to achieve that. Through the earlier entry of the eCMP in the TOSP, the robot can start its toe-off motion earlier, which helps avoiding the rear knee singularity and maximize step length and height $(\rightarrow$ high stairs). Therefore, we consider two eCMPs - one close to the heel and one close to the toe - instead of only one central eCMP for each foot. As before, the corresponding heel- and toe-VRPs $\left(\boldsymbol{r}_{v r p, H, i}\right.$ and $\left.\boldsymbol{r}_{v r p, T, i}\right)$ are $\Delta z_{v r p}$ above the heel- and toe-eCMPs. Instead of one central VRP in each foot, we now use both heel- and toe VRPs in each foot to first derive the preliminary DCM trajectory (dark blue lines in Fig. 5 (right)), assuming instantaneous transitions. As in [17], this is done via backwards recursion (see (8)), starting with the final previewed toe-VRP and iterating backwards in time over all heel- and toe-VRPs until the current one. That way, all future initial heel-to-toe DCMs $\left(\boldsymbol{\xi}_{\text {ini,HT,i }}\right)$ and initial toe-to-heel DCMs $\left(\boldsymbol{\xi}_{\text {ini,TH,1 }}\right)$ are computed. In (8) instead of $t_{\text {step }}$ we now use $\Delta t_{H T}=\alpha_{H T} t_{\text {step }}$ (heel-to-toe transition) and $\Delta t_{T H}=\left(1-\alpha_{H T}\right) t_{\text {step }}$ (toe-to-heel transition) as the duration of transition. The parameter $\alpha_{H T}$ may be chosen in the range of $0 \leq \alpha_{H T} \leq 1$ (here: $\alpha_{H T}=0.5$ ). Just like in Sec. III-A, the edges around the initial heel-to-toe-DCMs are now rounded via third order polynomial splines (turquoise curves in Fig. 5 (right)) to achieve a continuous VRP transition from toe to heel during double support. The initial and final double support DCM positions can be computed via

$$
\boldsymbol{\xi}_{\text {iniDS }, i}=\boldsymbol{r}_{v r p, T, i-1}+e^{-\frac{\Delta t_{D S, i n i}}{b}}\left(\boldsymbol{\xi}_{\text {ini }, H T, i}-\boldsymbol{r}_{v r p, T, i-1}\right)
$$

and

$$
\boldsymbol{\xi}_{\text {eoDS }, i}=\boldsymbol{r}_{v r p, H, i}+e^{\frac{\Delta t_{D S, e n d}}{b}}\left(\boldsymbol{\xi}_{\text {ini }, H T, i}-\boldsymbol{r}_{v r p, H, i}\right) .
$$

$\boldsymbol{\xi}_{\text {iniDS }, i}, \boldsymbol{\xi}_{e o D S, i}$ and the according DCM velocities as used as boundary conditions in (16) to compute the polynomial parameters for the double support phases. Unlike in Sec. IIIA, the single support DCM reference trajectory is not computed via exponential interpolation (9) but again via the polynomial interpolation (17) with $\boldsymbol{\xi}_{e o D S, i-1}$ as initial and $\boldsymbol{\xi}_{\text {iniDS,i } i}$ as final boundary condition in (16), resulting in the bright blue curves in Fig. 5 (right). The pink curves in Fig. 5 (right) illustrate the continuous VRP transitions during single support (from $\boldsymbol{r}_{v r p, H, i}$ to $\boldsymbol{r}_{v r p, T, i}$ ) and double support (from $\boldsymbol{r}_{v r p, T, i}$ to $\boldsymbol{r}_{v r p, H, i+1}$ ). Again, the actual DCM,
VRP and eCMP trajectories are three-dimensional in general (Fig. 5 shows a top view). As mentioned before, the use of the proposed polynomial interpolation does not guarantee feasibility of the commanded VRPs (corresponding CoP in base of support). Yet, for the chosen parameters $\alpha_{D S, \text { ini }}=0.5$ and $\alpha_{H T}=0.5$ we did not encounter any feasibility violation in simulations or experiments.

Figure 1 shows the simulated Atlas robot taking $70 \mathrm{~cm}$ steps, which was facilitated by the Heel-to-Toe trajectories described in this section. In this paper, we only consider toeoff during double support. Toe-off during single support is also conceivable but not explicitly covered here.

Table I summarizes which interpolation method is used for what controller during each phase.

\begin{tabular}{|l|l|c|c|}
\hline \multirow{2}{*}{ controller } & \multicolumn{2}{|c|}{ DCM interpolation method } \\
\hline \multicolumn{2}{|l|}{ discontinuous } & $\mathrm{x}$ & \\
\hline \multirow{2}{*}{ conti. DS } & double support & & $\mathrm{x}$ \\
\cline { 2 - 4 } & single support & $\mathrm{X}$ & $\mathrm{x}$ \\
\hline \multirow{2}{*}{ heel-to-toe } & double support & & $\mathrm{x}$ \\
\cline { 2 - 4 } & single support & & polynomial (17) \\
\hline
\end{tabular}

TABLE I

\section{WALKING STATE MACHINE}

We make use of a walking state machine (see Fig. 7) to keep track of and thoroughly switch between walking states and provide the DCM interpolators with their necessary inputs (such as previewed foot positions and the current time in state $t$ ). In total we distinguish between four states: Stance, Initial Transfer (IT), Single Support (SS), Double Support (DS). Stance can be seen as the robot idle state, where no walking trajectories are commanded and it is simply balancing in place. During Initial Transfer, the robot shifts its DCM from between its stance feet to the final double support DCM position $\boldsymbol{\xi}_{e o D S, 2}$ of the upcoming step (see Fig. 5). Single and Double Support state correlate to the phases described in Sec. III.

A timer associated to the walking state machine provides the exponential and polynomial interpolators (9) and (17) with the time in state $t$. It incrementally increases $t$, until the current walking state duration is reached $(t \in[0, T]$, where $\left.T \in\left\{T_{I T}, T_{S S}, T_{D S}\right\}\right)$, which triggers a state transition and resets $t(t=0)$. After each DS phase, the state machine checks, whether there are more steps to take (e.g. more elements in list of preplanned footstep positions) and accordingly decides to switch to stance or the next single support phase. 

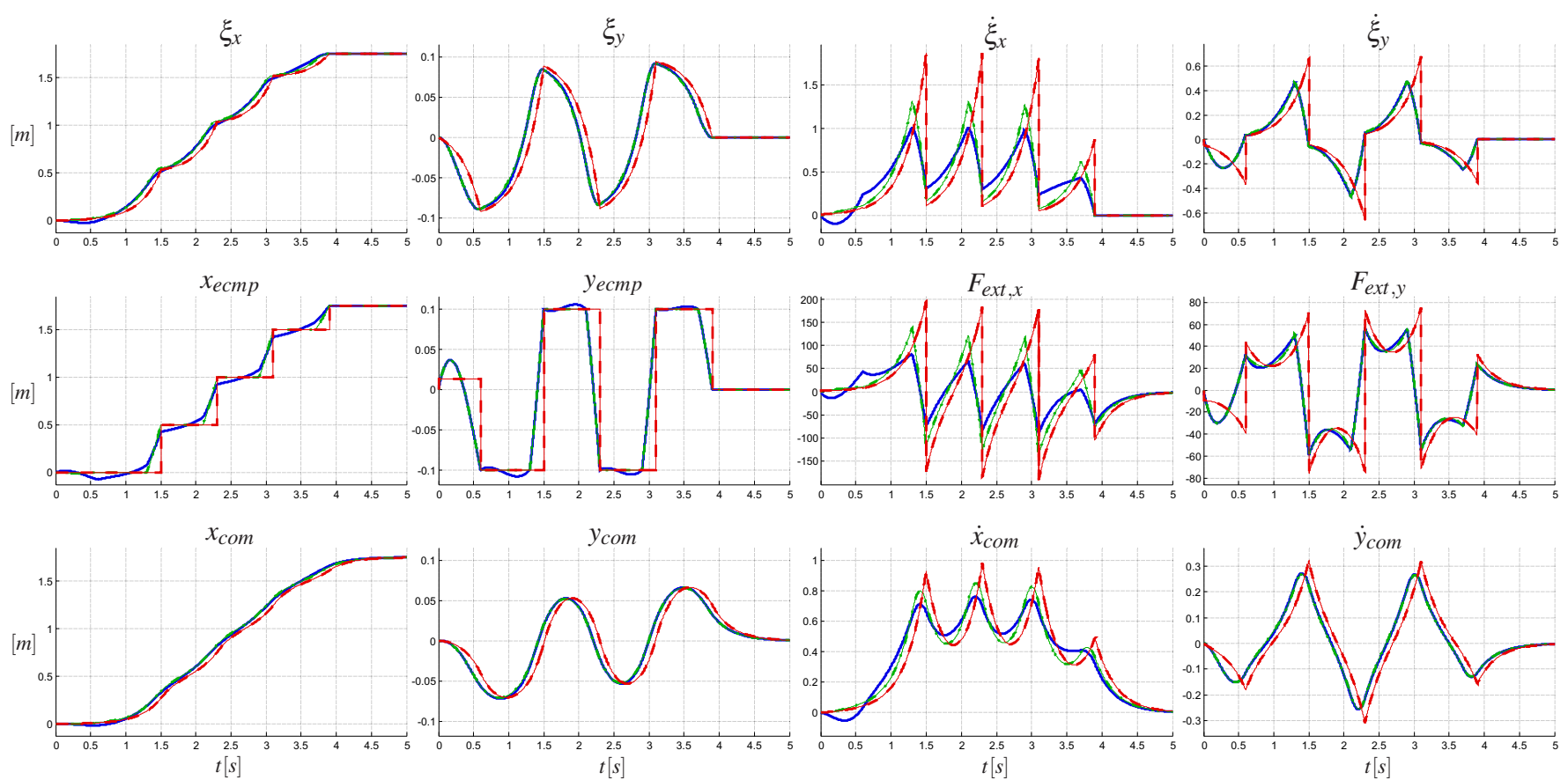

Fig. 8. Comparison of discontinuous (red dashed), Continuous Double Support (green dash-dotted) and Heel-to-Toe (blue solid) trajectory generators. Shown trajectories correspond to a walk with the following parameters: $t_{t e p}=0.8 s, t_{D S}=0.2 s$, step length $0.5 \mathrm{~m}$, step width $0.2 \mathrm{~m}$, foot length $0.15 \mathrm{~m}$

In our implementation, we distinguish between the list of preplanned footsteps (e.g. 100 preplanned footsteps for walking from one room to another) and the list of previewed steps. The latter contains only a number (in our implementations typically four, as in Fig. 5) of steps, which are considered for DCM reference trajectory generation. In the beginning of each single support phase the list of previewed steps is updated and all reference points (see Sec. III) for the current single support and upcoming double support are computed. Also the polynomial parameters (see Sec. III) for smooth transitions are pre-computed. For advanced applications (e.g. online foot-adjustment), these computations might be done every control cycle, otherwise this once-per-step precomputation is sufficient. Note that the index " 1 " always denotes the current (or hind) stance foot, whilst the indices " 2,3 ..." denote the next, second next and so on footsteps.

\section{DisCUSSION OF RESULTING DCM REFERENCE TRAJECTORIES}

Figure 8 shows a comparison of the discontinuous, Continuous Double Support (CDS) and Heel-to-Toe (HT) trajectory generators for a 4-step sample walk of a bipedal robot (find details of the walking task in the caption).

For the Continuous Double Support (CDS) and Heel-toToe (HT) trajectories derived in Sec. III, the force profiles are continuous (as compared to discontinuous force profiles for the original method [17]) and the maximum absolute of the leg-forces is strongly decreased, resulting in smaller minimum joint torques required for walking.

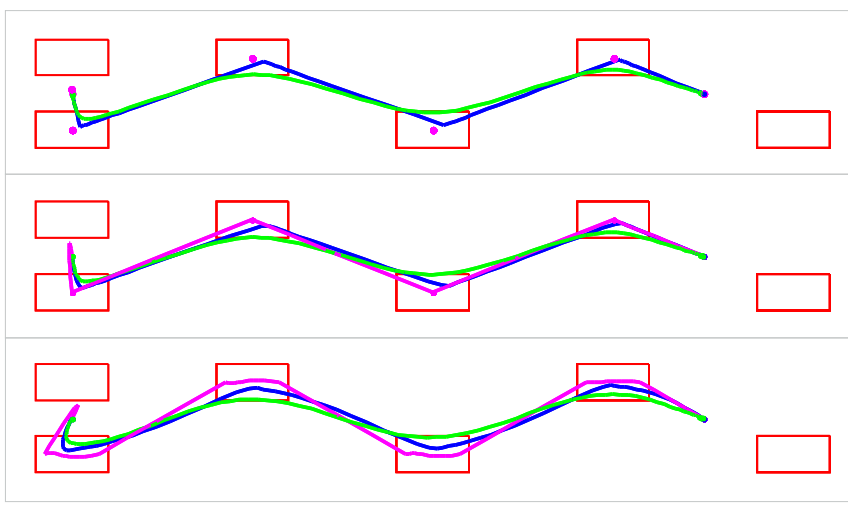

Fig. 9. Comparison (top view) of VRP (pink), DCM (blue) and CoM (green) reference trajectories. top: discontinuous [17], middle: Continuous Double Support (Sec. III-A), bottom: Heel-to-Toe (Sec. III-B) trajectories

The maximum DCM velocity (especially in sagittal direction $x$ ) is drastically reduced (see quantitative comparison in table II). As the term "Divergent Component of Motion" indicates, the DCM velocity is a measure for how fast one of the robot states (the DCM) is diverging. Although we make use of this divergence to allow the robot to dynamically walk ( $\rightarrow$ "controlled divergence"), it comes with the inherent danger of loss of control if the actual interactions of the robot system with its environment differs from the predicted ones. For instance in case of a delayed landing of the swing foot, the DCM may during single support overshoot out of the previewed (double support) support polygon, which may result in unrecoverable instability of the robot. Thus, 

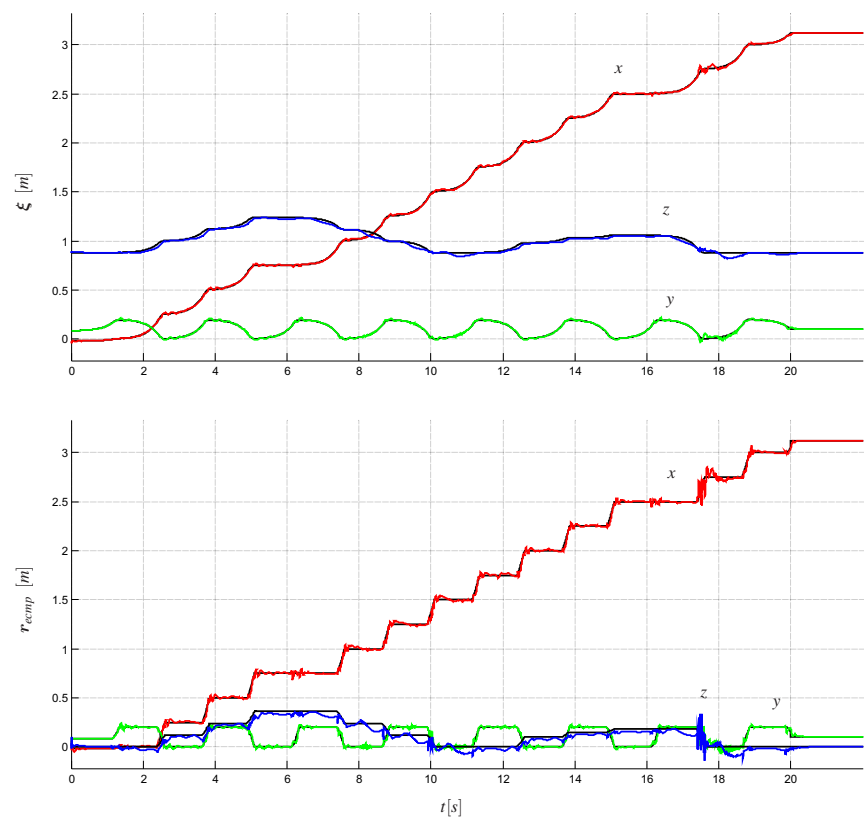

Fig. 10. OpenHRP3 simulation of humanoid robot TORO. Top: desired (black) and real DCMs, Bottom: desired (black) and real eCMPs

the lower DCM velocities observed for the CDS and HT trajectories decrease the risk of loss of control.

One can also observe lower maximum CoM velocities for the CDS and HT trajectories, which correlates to lower required joint speeds or higher maximum walking speed for a given maximum joint velocity.

Table II shows a quantitative comparison of the achieved sagittal forces, DCM velocities and CoM velocities.

\begin{tabular}{|l|l|l|l|l|l|l|}
\hline algorithm & \multicolumn{2}{|c|}{$F_{\text {ext,x }}[N]$} & \multicolumn{2}{c|}{$\xi_{x, \max }\left[\frac{m}{s}\right]$} & \multicolumn{2}{|c|}{$\dot{x}_{\text {com,max }}\left[\frac{\mathrm{m}}{s}\right]$} \\
& abs. & perc. & abs. & perc. & abs. & perc. \\
\hline discont. DS & 190 & $100 \%$ & 1.86 & $100 \%$ & 0.98 & $100 \%$ \\
\hline cont. DS & 136 & $71.6 \%$ & 1.31 & $70.4 \%$ & 0.85 & $86.7 \%$ \\
\hline cont. DS/HT & 90.2 & $47.5 \%$ & 1.01 & $54.3 \%$ & 0.76 & $77.6 \%$ \\
\hline
\end{tabular}

TABLE II

\section{Simulations, EXPERIMENTS AND TRIALS}

The proposed DCM reference trajectory generators from Sec. III (and the associated DCM feedback control (10)) were thoroughly tested in numerous simulations (both for DLR's humanoid robot TORO and IHMC's Atlas robot) and experimentally with IHMC's Atlas robot.

Figure 10 shows the desired and real (three-dimensional) DCM and eCMP trajectories for an OpenHRP3 [23] simulation in which TORO walks over a set of stairs (see Fig. 11). The 3D DCM trajectories are tracked accurately and the eCMP transitions are smooth during double support.

During the preparations for DARPA's Virtual Robotics Challenge (VRC), the Continuous Double Support (CDS) trajectory generator (Sec. III-A) was first developed and subsequently evolved into the Heel-to-Toe (HT) trajectory

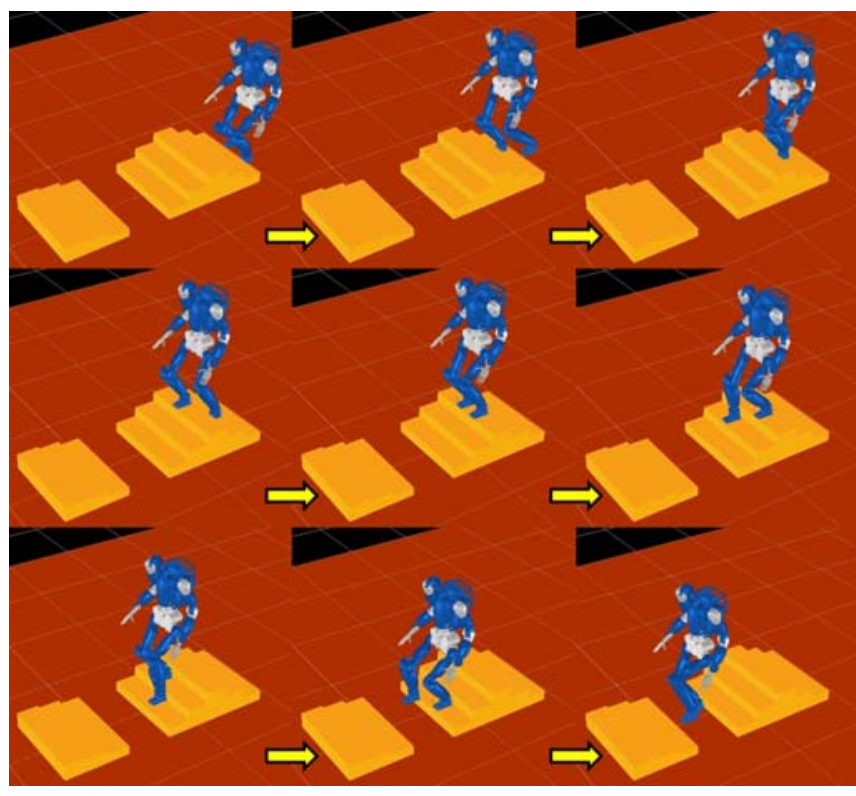

Fig. 11. OpenHRP3 simulation of DLR's bipedal humanoid TORO. Walking over a set of stairs of variable height. (step height differences: $[+12,+12,+12,-12,-12,-12,+10,+5,+3,-18] \mathrm{cm})$

generator (Sec. III-B). Based on the latter, the simulated Atlas robot achieved a maximum step length of up to $70 \mathrm{~cm}$ (see Fig. 1) in both IHMC's simulation environment [10] and the official VRC simulator Gazebo [24].

The algorithms described in this paper were also used during IHMC's participation in the DARPA Robotics Challenge Trials 2013 [25]. Amongst others, IHMC's Atlas robot accomplished to get full marks for the terrain task ( $\rightarrow$ ramps, steps, stairs, inclined stairs). Figure 12 shows an experimental result of Atlas walking on flat ground while using the Continuous Double Support (CDS) trajectory generator (from Sec. III-A). The plot shows a series of seven steps after which Atlas comes to a stop. It displays the estimated eCMP, DCM and CoM trajectories in $x$ (forward) and $y$ (sideward) direction. We use the term "estimated" here, as these quantities were not measured directly but computed from joint torques and estimated via a state observer (Kalman filter). The eCMP transition during double support is continuous, resulting in continuous leg force profiles.

\section{CONCLUSION AND FUTURE WORK}

In this paper, we presented two DCM trajectory generators (the Continuous Double Support (CDS) and Heel-to-Toe (HT) trajectory generator) which build up on the method for three-dimensional DCM trajectory generation introduced in [17] and can easily be computed in real-time. They lead to continuous leg force transitions during double support phase and facilitate toe-off motion of a walking robot. The performance of the proposed methods was tested in numerous simulations (both for IHMC's Atlas robot and DLR's humanoid robot TORO) and in experiments on IHMC's Atlas 

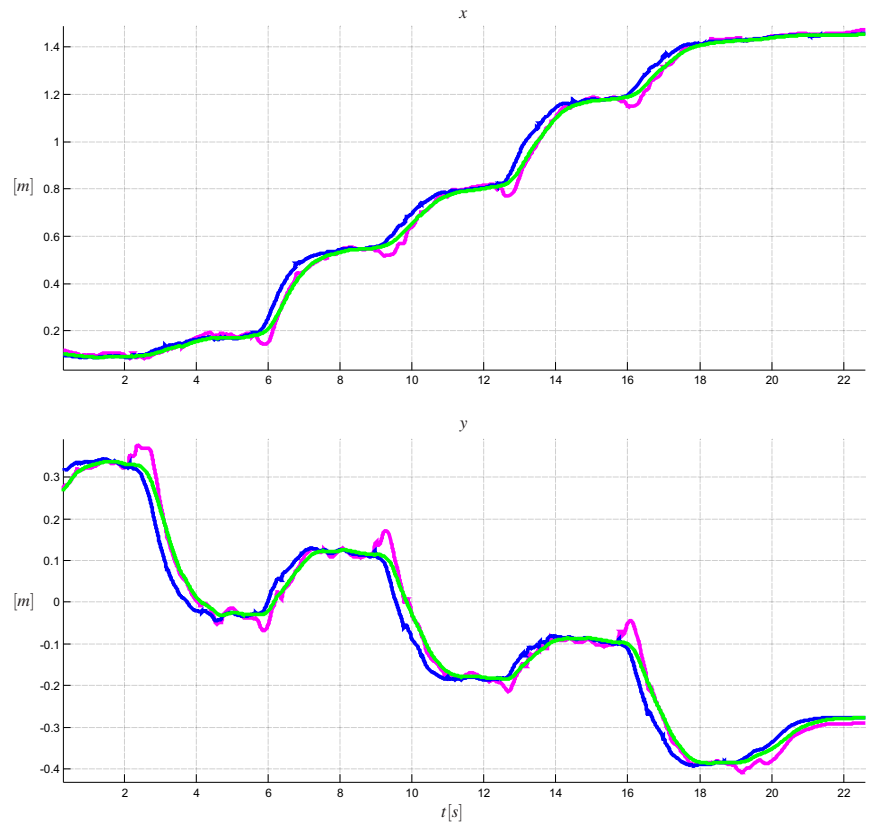

Fig. 12. Experimental results of IHMC's Atlas robot walking during DRC Trials. Pink: estimated eCMP, blue: est. DCM, green: est. CoM

robot. Future work includes enhancements of the algorithms for the use in the DARPA Robotics Challenge (e.g. for toeoff motion to step onto large obstacles) and the embedding of the generators into DLR's continuous replanning framework ( $\rightarrow$ e.g. for online responsive joystick steering).

\section{ACKNOWLEDGEMENTS}

This research is partly supported by the Initiative and Networking Fund of Helmholtz Association through a Helmholtz Young Investigators Group (Grant no. VH-NG-808).

The first author wants to cordially thank Dr. Jerry Pratt and his colleagues at IHMC's robotics lab for welcoming him for a three months research visit during DARPA's Virtual Robotics Challenge (VRC) in 2013.

\section{REFERENCES}

[1] T. Takenaka, T. Matsumoto, and T. Yoshiike, "Real time motion generation and control for biped robot, 1st report: Walking gait pattern generation," in IEEE/RSJ Int. Conf. on Intelligent Robots and Systems, 2009, pp. 1084-1091.

[2] B. Stephens and C. Atkeson, "Push recovery by stepping for humanoid robots with force controlled joints," in IEEE-RAS Int. Conf. on Humanoid Robots, 2010, pp. 52-59.

[3] P.-B. Wieber, "Trajectory free linear model predictive control for stable walking in the presence of strong perturbations," in IEEE-RAS Int. Conf. on Humanoid Robots, 2006, pp. 137-142.

[4] A. Herdt, N. Perrin, and P.-B. Wieber, "Walking without thinking about it," in IEEE/RSJ Int. Conf. on Intelligent Robots and Systems, 2010, pp. 190-195.

[5] S. Kajita, M. Morisawa, K. Miura, S. Nakaoka, K. Harada, K. Kaneko, F. Kanehiro, and K. Yokoi, "Biped walking stabilization based on linear inverted pendulum tracking," in IEEE/RSJ Int. Conf. on Intelligent Robots and Systems, 2010, pp. 4489-4496.

[6] Y. Choi, D. Kim, Y. Oh, and B.-J. You, "Posture/walking control for humanoid robot based on kinematic resolution of com jacobian with embedded motion," IEEE Transactions on Robotics, vol. 23, no. 6, pp. 1285-1293, 2007.
[7] S. Kajita, F. Kanehiro, K. Kaneko, K. Fujiwara, K. Harada, K. Yokoi, and $\mathrm{H}$. Hirukawa, "Biped walking pattern generation by using preview control of zero-moment point," in IEEE Int. Conf. on Robotics and Automation, 2003, pp. 1620-1626.

[8] Y. Zhao and L. Sentis, "A three dimensional foot placement planner for locomotion in very rough terrains," in IEEE-RAS Int. Conf. on Humanoid Robots, 2012, pp. 726-733.

[9] "Atlas - the agile anthropomorphic robot." [Online]. Available: http://www.bostondynamics.com/robot_Atlas.html

[10] "Simulation construction set - ihmc's simulation environment for humanoid robots." [Online]. Available: http://ihmc.us/groups/scs/

[11] A. L. Hof, "The 'extrapolated center of mass' concept suggests a simple control of balance in walking," Human Movement Science, vol. 27, pp. 112-125, 2008.

[12] T. Koolen, T. D. Boer, J. Rebula, A. Goswami, and J. E. Pratt, "Capturability-based analysis and control of legged locomotion. part 1: Theory and application to three simple gait models," The Int. Journal of Robotics Research, vol. 31, no. 9, pp. 1094-1113, 2012.

[13] J. Englsberger, C. Ott, M. A. Roa, A. Albu-Schäffer, and G. Hirzinger, "Bipedal walking control based on capture point dynamics," in IEEE/RSJ Int. Conf. on Intelligent Robots and Systems, 2011, pp. $4420-4427$.

[14] J. Englsberger and C. Ott, "Integration of vertical com motion and angular momentum in an extended capture point tracking controller for bipedal walking," in IEEE-RAS Int. Conf. on Humanoid Robots, 2012, pp. 183-189.

[15] M. Morisawa, S. Kajita, F. Kanehiro, K. Kaneko, K. Miura, and K. Yokoi, "Balance control based on capture point error compensation for biped walking on uneven terrain," in IEEE-RAS Int. Conf. on Humanoid Robots, 2012, pp. 734-740.

[16] K. Seo, J. Kim, and K. Roh, "Towards natural bipedal walking: Virtual gravity compensation and capture point control," in IEEE/RSJ Int. Conf. on Intelligent Robots and Systems, 2012, pp. 4019-4026.

[17] J. Englsberger, C. Ott, and A. Albu-Schäffer, "Three-dimensional bipedal walking control using divergent component of motion," in IEEE/RSJ Int. Conf. on Intelligent Robots and Systems, 2013, pp. 2600-2607.

[18] J. Pratt, J. Carff, S. Drakunov, and A. Goswami, "Capture point: A step toward humanoid push recovery," in IEEE-RAS Int. Conf. on Humanoid Robots, 2006, pp. 200-207.

[19] A. D. Kuo, "Energetics of actively powered locomotion using the simplest walking model," Journal of Biomechanical Engineering, vol. 124, pp. 113-120, 2002.

[20] Y. Ogura, K. Shimomura, H. Kondo, A. Morishima, T. Okubo, S. Momoki, H. ok Lim, and A. Takanishi, "Human-like walking with knee stretched, heel-contact and toe-off motion by a humanoid robot," in IEEE/RSJ Int. Conf. on Intelligent Robots and Systems, Oct 2006, pp. 3976-3981.

[21] M. B. Popovic, A. Goswami, and H. Herr, "Ground reference points in legged locomotion: Definitions, biological trajectories and control implications," The Int. Journal of Robotics Research, vol. 24, no. 12, pp. 1013-1032, 2005.

[22] M. Vukobratovic and Y. Stepanenko, "On the stability of anthropomorphic systems," Mathematical Biosciences, vol. 15, pp. 1-37, 1972.

[23] F. Kanehiro, K. Fujiwara, S. Kajita, K. Yokoi, K. Kaneko, H. Hirukawa, Y. Nakamura, and K. Yamane, "Open architecture humanoid robotics platform," in IEEE Int. Conf. on Robotics and Automation, 2002, pp. 24-30.

[24] "Gazebo - a multi-robot simulator for outdoor environments." [Online]. Available: http://gazebosim.org/about.html

[25] "Darpa robotics challenge trials 2013." [Online]. Available: http://www.theroboticschallenge.org/ 